\title{
La vejezy el deporte como objeto de representaciones sociales The old age and the sport like object of social representations
}

\author{
*Luis Javier Ruiz Cazorla, **José Luis Chinchilla Minguet y***Manuel Ruiz Cazorla \\ *IES Cánovas del Castillo, **Universidad de Málaga, ***IES Nuevo de Benamocarra (España)
}

\begin{abstract}
Resumen: En el presente trabajo analizamos la función social de la Educación Física y el deporte respecto a las personas mayores. Se trata de una relación mediatizada por las representaciones sociales que los agentes implicados poseen sobre la vejez, el cuerpo, la salud y el deporte, no exentas de estereotipos y prejuicios que condicionan sus prácticas sociales. Constituye un problema social y a la vez un reto para las instituciones erradicar las prácticas sociales de exclusión a que dan lugar. Para ello resultan imprescindibles dos pasos. En primer lugar denunciar los contenidos de las representaciones sociales sobre la vejez y las actividades físico-deportivas que favorecen la exclusión social, especialmente desde el Sistema Educativo y los medios de comunicación. En segundo lugar promover los medios materiales y condiciones estructurales necesarias para el cambio social.
\end{abstract}

Palabra clave: Vejez, deporte, educación física, representación social, función social.

Abstract: In the present work we analyzed the social function of the Physical Education and the sport with respect to the elderly people. One is a relation hyped by the social representations that the implied agents own on the old age, the body, the health and the sport, nonfree of stereotypes and prejudices that condition their social practices. It constitutes a social problem and simultaneously a challenge for institutions to eradicate the social practices of exclusion to that they give rise. For it two steps are essential. In the first place to denounce the contents of the social representations on the sport old age and activities physical that favor the social exclusion, especially from the Educative System and mass media. Secondly to promote the average materials and necessary structural conditions for the social change.

Key words: Old age, sport, physical education, social representation, social function.

\section{Introducción}

La vejez es una categoría social que trata de conceptuar una etapa de la vida humana, un proceso que denominamos envejecimiento caracterizado por una serie de cambios biológicos y psicosociales. Como toda categoría social, es una conceptualización cultural y por tanto relativa. Así, la Organización Mundial de la Salud(OMS) considera la edad de 65 años como el límite inicial de la vejez, mientras que la Organización de las Naciones Unidas (ONU) rebaja su comienzo a los 60 años de edad. En cualquier caso estas delimitaciones están sometidas a numerosas variables culturales, étnicas y sobre todo económicas, que hacen que tanto en las expectativas de vida como en las condiciones de llegada a la vejez se produzcan grandes diferencias a lo largo y ancho del planeta, e incluso dentro de un mismo país o zona geográfica.

Centrándonos ya en nuestro contexto social y en las relaciones entre vejez y actividad físico deportiva, nos encontramos ante un campo de la vida social cada vez más complejo, sometido a un cambio constante, dondeintervienen múltiples factores, culturales, tecnológicos, económicos y políticos. Desde una perspectiva psicosocial, se configura como un campo representacional en el que interaccionan representaciones sociales (RSs) de diferentes objetos como la vejez, el cuerpo, la salud y el deporte. Dicho campo representacional genera a su vez determinadas prácticas sociales como la discriminación y la exclusión, a las que las administraciones y sus responsables están obligados a dar respuesta, especialmente aquellos que desempeñamos nuestra labor en el ámbito educativo.

Se necesita, pues, realizar una reflexión y esfuerzo conjunto y continuado de instituciones, investigadores y profesionales relacionados con las actividades físicas y deportivas sobre sus propias RSs acerca de la vejez y el deporte, para difundir, a través del sistema educativo, de la práctica cotidiana y de los medios de comunicación, los actuales conocimientos científicos sobre el deporte, la vejez, el cuerpo y la salud, y fomentar en definitiva un desarrollo más integral, justo y saludable del ser humano.

En el presente trabajo reflexionamos y proponemos la teoría de las RSs como un marco teórico y metodológico pertinente para el estudio

\footnotetext{
Fecha recepción: 15-01-10 - Fecha envío revisores: 15-01-10 - Fecha de aceptación: 15-01-10 Correspondencia: Luis Javier Ruiz Cazorla

C/Primavera 2, $4^{\circ} \mathrm{B}$,

29700, Vélez-Málaga (Málaga)

E-mail: ljulises@hotmail.com
}

de las relaciones entre la vejez y las actividades físico deportivas, así como de los problemas sociales que se dan en este ámbito. La propuesta se enmarca en la línea de investigación Ciencias Sociales aplicadas al Deporte y la Educación Física que actualmente desarrollamos en el grupo de investigación HUM 564 Educación Física y Deporte. Formación del Profesorado en Andalucía.

\section{Planteamiento del problema}

La cuestión de las relaciones entre vejez y actividades físico deportivas puede abordarse desde diferentes posiciones que presuponen a su vez diferentes teorías sobre el envejecimiento, tanto desde la óptica biológica como de la psicosocial, que se encuentran en el núcleo de sus RSs. Estas teorías no son neutrales, sino que parten a menudo de creencias y estereotipos con bases ideológicas, que determinan las funciones sociales del deporte, que responden a un modelo de sociedad, a intereses económicos o políticos, y que condicionan tanto la investigación como las prácticas sociales, dando lugar a fenómenos de marginación y exclusión social. El mismo hecho de que en la terminología de eventos como el presente, la expresión de 'persona mayor' trate de desplazar al concepto más popular de ‘viejo’ es una muestra del intento institucional de combatir a través del lenguaje, mediador fundamental de las RSs, contra los prejuicios sociales acumulados históricamente por este último término.

La sociedad encara al anciano y lo identifica de maneras diferentes. En la percepción estereotipada de la realidad que caracteriza al ser humano, algunos tienen una imagen preconcebida de los ancianos haciendo ganchillo, sentados en el banco de un parque viendo pasar el tiempo, con los cabellos blancos, sedentarios, como mucho jugando al dominó o a la petanca, o fatalmente enfermos. Otros, sin embargo, encaran a la persona mayor como alguien que puede estar jubilada o trabajando, viajando o estudiando, realizando actividades en la naturaleza, ciclismo o natación, y sexualmente activa. Son diferentes RSs de un mismo objeto social, o mejor dicho de un mismo campo representacional ya que, como indicamos más arriba, hablamos de la interrelación de varias RSs (cuerpo, salud, vejez, actividad física, deporte).

En cualquier casoy desde nuestra perspectiva, el debate del problema se debe centrar, más que en el grado de veracidad o falsedad de los estereotipos, en la utilización que se hace de los mismos en las prácticas sociales, que es la que determina su conversión en prejuicios. Defender que los estereotipos son falsos porque se haya encontrado uno que no se corresponda con la realidad tiene tan poco fundamento como afirmar 
que son siempre verdaderos porque se haya encontrado uno que lo sea. Sería el rasgo de rigidez del estereotipo el aspecto clave a analizar, entendiéndolo como su resistencia al cambio ante las informaciones que lo contradicen (Cano Gestoso, 1993). Este factor cobra gran importancia en una sociedad como la postmoderna, caracterizada por la rapidez de los cambios tecnológicos y sociales, así como por el dinamismo en la circulación de las informaciones a través de los medios de comunicación social.

El estereotipo de persona mayor como ser enfermo, incapaz y dependiente está cambiando en nuestra sociedad, abriéndose paso una nueva identidad para el anciano. En el pasado la vejez estaba ligada a la invalidez o incapacidad de producir y una preocupación social era saber quien cuidaría de los obreros envejecidos o enfermos. La vejez era vista como una «antecámara» de la muerte, caracterizada por las canas, las dificultades motrices y perceptivas, o vinculada a la idea de incapacidad laboral y sexual, dando lugar a prejuicios que semanifestaban en prácticas sociales de exclusión, ridiculización y negación.

\section{Marco teórico para la investigación social del deporte}

Cualquier investigación sobre la realidad social exige, desde nuestro punto de vista, definir el marco científico que se ocupa de este tipo de fenómenos, las ciencias que los estudian y las teorías más importantes desarrolladas hasta el momento. Desde esta perspectiva, el modelo teórico señala dónde hay que buscar y qué ha de buscarse, ordena y selecciona datos, muestra analogías y destaca diferencias (Blanchard \& Cheska, 1986)

En el ámbito de la Sociología del Deporte, García Ferrando (1990) ha planteado la necesidad de considerar el pluralismo del pensamiento sociológico y el deporte a la hora de determinar el modelo teórico para este tipo de investigaciones. La misma complejidad de la realidad social impide que unúnico modelo teórico pueda dar respuesta a las necesidades que su estudio implica. Ningún modelo teórico puede aspirar a conocer toda la realidad social, a ser completamente objetivo y omnicomprensivo, por ello se postula la adopción de un pluralismo cognitivo para los fenómenos sociológicos. Hacen falta diversidad de modelos teóricos y de perspectivas metodológicas para tratar de describir y explicar una realidad social que es multidimensional, contradictoria y ambigua.

La acción social está condicionada por la estructura social, pero también por los procesos cognitivos que intervienen en el comportamiento de las personas. Por ello, cualquier estudio que trate de explicar la función social del deporte deberá tener en cuenta las teorías que tratan de explicar dicha acción desde una perspectiva tanto sociológica como psicológica(Ruiz Cazorla, 2008). Estas teorías, entre las que destacamos la teoría de las representaciones sociales, cumplen igualmente la importante función de ofrecer al investigador los instrumentos conceptuales necesarios para abordar el estudio sistemático y científico de la dimensión social del deporte.

Una cuestión previa para entender la forma en que la sociedad afronta la actividad físico deportiva de las personas mayores es precisamente la conceptualización del deporte que se encuentra en el núcleo de sus RSs. Este aspecto es especialmente relevante en los ámbitos científicos y especializados, ya que va a determinar la percepción, valoración y forma de abordar el problema por parte de educadores, investigadores y demás agentes sociales con responsabilidades en la materia.

La principal dificultad que se encuentra a la hora de conceptuar el deporte es la diversidad de definiciones existentes acerca del mismo, diversidad que nace sobre todo de las diferentes perspectivas de análisis de las que parte cada autor. Hasta el momento ningún autor ha podido definir en qué consiste el deporte de una manera completa, concluyente y que sea unánimemente aceptada. Ya sea desde una perspectiva social, cultural o exclusivamente motriz, todas las definiciones dejan de lado algún rasgo o elemento imprescindible a ojos de otros analistas. La diversidad de significados del deporte unida a su globalización son los más claros indicadores de su compleja inserción en la sociedad postmoderna, pero también de su propia evolución histórica.
En la actualidad el deporte constituye un fenómeno diverso y complejo casi imposible de delimitar. Como ya afirmó José María Cagigal (1981), cada día que pase resultará más difícil la definición del deporte, pues aparte de encontrarse en un cambio constante, ha ampliado y sigue ampliando su significado, y todo al ritmo que marca la propia evolución social y cultural. En esta idea coincide también García Ferrando (1990), para quien el deporte, detrás de su aparente simplicidad esconde una enorme complejidad social y cultural. Por ello este autor afirma que una definición del 'deporte' debe ser amplia y flexible, de forma que destaque sus elementos esenciales y sea inclusiva en lugar de exclusiva.

En cualquier caso, la falta de una definición de deporte acorde con los planteamientos de las ciencias sociales ha dado lugar a divergencias en cuanto a las circunstancias concretas que propiciaron la aparición del deporte, el peso que hay que conceder a las mismas o la función social y política que atribuyen al deporte en el mundo contemporáneo. Aunque no es el momento ni el lugar de hacer una revisión de la conceptualización del deporte, sí nos interesa destacar la idea de que las teorías explicativas tanto del origen como de las funciones del deporte en la sociedad se han decantado mayoritariamente por una postura idealista, fruto de una concepción heredada esencialmente del olimpismo moderno.

En el marco de esta concepción heredada, ligada a una determinada interpretación de la historia y de la cultura, el exagerado énfasis del olimpismo en las propiedades formativas y educativas del deporte para la juventud es un aspecto clave que haciéndose extensible del olimpismo al resto del deporte, ha influido notablemente en una RS del mismo vinculada a una imagen del cuerpo y de la estética, y a una prevalencia de cualidades físicas (recordemos el famoso lema citius, altius, fortius) que no favorece precisamente la inclusión de las personas mayores dentro del espectro social del deporte y el olimpismo:

«El agonismo litúrgico heleno es durante siglos la encarnación de las máximas aspiraciones estéticas y morales de un puebloy una cultura inigualable que, dotados del soplo divino de la inspiración consiguieron como dato cualificador de sus virtudes estéticas el inalcanzable don del equilibrio. La aretéy la calocagathia son patrones olímpicos que guían durante siglos a la juventud griega de los mejores tiempos.» (Durantez, 2002, p.17)

Un rasgo imprescindible a incluir en la conceptualización del deporte desde la perspectiva del investigador que aborda su dimensión social debería ser el de acción social. Los rasgos de codificación e institucionalización propuestos entre otros autores por Parlebás (1988) apuntan en este sentido al establecer nexos de interacción del deporte con el medio social. La codificación se relaciona con el sistema denormas y valores de cada sociedad, mientras que el rasgo institucional permite insertar al deporte en el marco de la estructura social. Sin embargo, dichos rasgos no han sido desarrollados en relación con los individuos o protagonistas del acto social desde una perspectiva que permita abordar los procesos cognitivos y sociales implícitos en el deporte.

Por ello, y teniendo en cuenta la reflexión anterior, proponemos partir de la siguiente definición de deporte que además de incluir rasgos esenciales ya propuestos por otros autores, incorpora los referidos a su dimensión psicosocial:

El deporte constituye una acción social desarrollada en un marco institucional, con o sin presencia de público, en la que uno o varios actores interaccionan mediante situaciones motrices codificadas de forma competitiva, generando consecuencias tanto en los individuos como en la estructura social. (Ruiz Cazorla, 2008)

En esta definición podemos destacar tres rasgos clave que según el autor conforman el deporte como una parcela de la realidad social permitiendo abordar su análisis empírico:

Acción social: la acción se puede definir como un proceso en el que las personas persiguen la consecución de metas que les son gratificantes (Garrido \& Álvaro, 2007). Interpretar el deporte como una acción social no implica entenderlo como una mera propiedad individual o reducida a sus protagonistas más directos, los deportistas. Su análisis supone establecer un vínculo de unión entre la cultura, el sistema social y la personalidad, que afecta a todas las personas implicadas en el mismo, deportistas, entrenadores, jueces, organizadores y aficionados. 
Marco institucional: aplicado al estudio del deporte este concepto sitúa los deportes en el contexto de las subestructuras que componen la estructura social. El deporte como institución, es una estructura social visible, con una organización jurídica y material, y un conjunto de conductas que se han de seguir apoyadas por normas y sanciones. Existe una dependencia e interacción entre las diferentes instituciones de una sociedad, pues éstas juntamente con los modelos de conducta que prescriben dentro de su ortodoxia, están configuradas por los valores y normas de la sociedad global.

Codificación competitiva: la reglamentación del deporte en forma de competición es un rasgo esencialmente cultural ya que está directamente conectado con el sistema denormas y valores dela sociedad. En el deporte las reglas cumplen la función que en la sociedad desempeña la norma social, un orden de valores orientativos que sirve para regular y definir el desarrollo de comportamientos comunes, a los que otorga cierto grado de legitimidad y consentimiento. La aplicabilidad de la norma está asegurada por las expectativas de sanciones positivas, así como por el miedo o la prevención a las negativas, lo que es consecuencia del grado de predominio de las costumbres de cada época y del nivel de interiorización de reglas o pautas a lo largo del proceso de socialización.

Por otro lado, público y afición constituyen un factor determinante, ya que marcan la frontera entre deporte praxis y deporte espectáculo, una frontera social no exenta de polémica y contradicciones. El deporte espectáculo por ejemplo introduce variables culturales, políticas y económicas que generan sustancialmente las RSs del deporte, y con ello las atribuciones, expectativas, actitudes y comportamientos de los agentes sociales implicados en cualquier acción deportiva.

La vejez y el deporte como objeto de representaciones sociales

La teoría de las RSs constituye una valiosa herramienta de investigación dentro y fuera del ámbito de la psicología social porque ofrece un marco explicativo acerca de los comportamientos de los grupos sociales estudiados que no se circunscribe a las circunstancias particulares de la interacción, sino que trasciende al marco cultural y a las estructuras sociales más amplias como, por ejemplo, las estructuras de poder y de subordinación (Araya Umaña, 2002).

Entre las principales funciones de las RSs está la de orientar el comportamiento de los individuos en el medio social, actuando como sistemas de regulación de las relaciones sociales. Regulan tanto la interacción social como el modo en que analizamos e interpretamos nuestro entorno, pues mediatizan nuestras relaciones con los objeto que constituyen el eje de la interacción social y a la vez son un instrumento de comprensión del ambiente social (Cerrato \& Villarreal, 2007). Las RSs no pueden entenderse como meras reproducciones sino, por el contrario, como reconstrucciones o recreaciones realizadas a partir de la experiencia de los actores sociales y su contexto cultural. Los mismos son sistemas cognitivos con una lógica y un lenguaje propio que se adquieren y modifican por la tradición, la educación y la comunicación social (Jodelet, 1984).

Laemergenciadeuna RS seve favorecidaporuna serie decondiciones (Moliner, 1996) que determinan al mismo tiempo, las preguntas que deben formularse al inicio de todo estudio:

- El objeto social al que se refiere debe ser polimorfo en el sentido en que puede aparecer bajo diferentes formas a la vez que permite un interjuego entre los diversos grupos sociales involucrados. La idea de envejecimiento diferencial según género, etnia, cultura, ocupación, entorno ambiental, etc,

- Se da en grupos sociales, es decir, son producidas y generadas colectivamente. Se pueden distinguir dos tipos de configuración según la relación que un grupo tiene con el objeto: a) estructural: concierne a grupos eníntima vinculación con el objeto, como es el caso del grupo de personas mayores estructurado en torno al objeto 'vejez' yb) coyuntural: supone grupos preexistentes que se encuentran confrontados a un objeto nuevo y problemático.

-Los interjuegos Existen factores que originan una RS: la identidad y la cohesión social. Cuando un individuo define su identidad en función de las RSs creadas colectivamente, refuerza al mismo tiempo la existencia del grupo como entidad social. En las configuraciones estructurales como es el caso de la vejez, el objeto se ubica en el centro del grupo de personas mayores, contribuyendo la identidad de sus miembros.

-La dinámica social. Se consideran las relaciones que mantiene el grupo frente a un objeto con respecto a las relaciones que otros grupos sociales tienen frente a dicho objeto. Desde esta perspectiva surgen dos posibilidades. Por un lado podría hacerse el estudio de las RSs de la vejez en grupos socialmente considerados como «no viejos» (niños, jóvenes, etc,). Aquí el grupo de la RS sería el de los 'no viejos’ mientras que el ‘otro’ social lo constituirían las personas mayores. Y por otro lado podría plantearse la situación inversa: estudiar las RSs de la vejez en las personas mayores, donde ellas mismas conformarían el grupo que ‘se representa’ y el ‘otro’ social estaría constituido por los «no viejos».

Función social del deporte respecto a las personas mayores

Hablar sobre las funciones sociales del deporte remite a una de las corrientes que más ha influido en la Sociología y en la Psicología Social, el funcionalismo estructural. La función es el concepto clave de esta teoría que explica los fenómenos sociales en términos de las funciones que éstos cumplen para el mantenimiento del orden social.Las funciones manifiestas serían las que resultan intencionales y conocidas para las personas que participan en dicha institución. Serían las prácticas que los actores conocen y persiguen de forma consciente. En el caso del deporte para las personas mayores podrían ser funciones manifiestas favorecer la inclusión social, mejorar....etc. Las funciones latentes serían las consecuencias no intencionales o no reconocidas por las instituciones o por la sociedad. Algunos ejemplos serían la influencia de factores como la ideología del éxito deportivo o el concepto de estética corporal en prácticas sociales de discriminación o exclusión social de determinados grupos que como el de personas mayores no responden a las pautas marcadas por estas ideologías.

Desde una perspectiva institucional, los actuales estados liberales y democráticos han atribuido múltiples funciones-beneficios al deporte conel fin de justificar suactuación en materia deportiva. Dicha intervención ha sido criticada por algunos autores como sospechosa de responder meramente a intereses políticos y económicos. Un ejemplo de estas críticas es la valoración hecha por el escritor Rafael Sánchez Ferlosio (1997)en un artículo de prensa titulado «El deportey el Estado» publicado en el diario 'El País', donde manifestaba lo siguiente:

«...no deja de ser cierto que el liberalismo puede encarecer los altísimos valores del deporte agónico para las sociedades de mercado libre, ilustrándolos con toda su consabida retahila de virtudes: la voluntad de autoafirmación y autorrealización, el afán de superación, la aspiración a la excelencia, el ardor competitivo, el amor por el trabajo, el espíritu de sacrificio, la impavidezy resistencia ante el esfuerzo y el dolor... todas ellas, en fin, puras y simples perversiones funcionales comunes a las culturas helénica y cristiana o tomadas de la una o de la otra.»

Esta perspectiva crítica del deporte ha sido desarrollada por diferentes autores, aportando claves que nos pueden ayudar a interpretar contenidos de las RSs del deporte que podrían contribuir a provocar situaciones de exclusión y desigualdad social. Brohn (1978) por ejemplo, apunta una serie de reflexiones que pueden ayudar a comprender las relaciones entre la vejezy el deporte a partir de los contenidos ideológicos implícitos en los contenidos de sus RSs. Dicho autor parte de la teoría de que la forma mercantil es la forma dominante de los intercambios orgánicos en una sociedad, que ejerce una influencia decisiva sobre todas las manifestaciones de la vida, imprimiendo su estructura a toda la conciencia del hombre. Para este autor, existe una profunda analogía estructural entre la organización y funcionamiento de un tipo de sociedad dada y una actividad socializada que desde el principio se impregna de estefuncionamiento: el rendimiento, como consecuenciadelapersecución de la ganancia. Este condicionamiento viene dado por una serie de rasgos que posee el deporte, especialmente por la competición, que para Brohm es la esencia misma del deporte.

Como agente de categorización social el deporte consagra el valor de la jerarquía por medio de la competición. Representaría una importante base ideológica desde la que se trata de justificar la situación de estatus 
y privilegios de la élite social. El más fuerte es quien gana. Y hay que tener en cuenta que la jerarquía es el principio sobre el que descansa todo el cuerpo social, es decir, el escalonamiento de los individuos y de los grupos dentro de la pirámide social en función de su posición de clase. El deporte, en cuanto competencia, clasificación y selección, contribuye a perpetuar la jerarquía que garantiza la estabilidad social, basada precisamente en la selección y la clasificación de los individuos en clases sociales. La sociedad es vertical, y, aparentemente, la jerarquización se realiza en función del mérito. Cada uno asciende en función de su trabajo y su ganancia, y así trata de parecerlo en el deporte, donde la competición garantiza el triunfo del mejor. Sin embargo este principio de jerarquía oculta para los marxistas su contenido profundo: la desigualdad real de los individuos. En este contexto, parecería lógico que las personas que han salido de la cadena productiva pierdan reconocimiento y sufran la exclusión social.

\section{Conclusiones}

El estudio empírico de la función social de las actividades físicas y deportivas es una condición que consideramos imprescindible para poder comprender el papel del deporte en la sociedad actual. Dicho estudio, que pasa por el de sus RSs, genera reflexiones e interrogantes cuyas respuestas deberían ser tenidas en cuenta por todos los que de una forma u otra están relacionados con el deporte, políticos, periodistas, educadores, y muy especialmente los dirigentes de las instituciones deportivas, si realmente pretenden hacer del mismo un hecho social y cultural que responda a las necesidades y valores del mundo actual.

En cualquier caso, nos encontramos ante una perspectiva de análisis que en absoluto pretende la elaboración de teorías definitivas, sino más bien proponer una línea de investigación, un marco conceptual y metodológico desde el que abordar la dimensión social del deporte, abriendo ideas al debate y a la revisión en su caso, que contribuyan al conocimientoy a la comprensión deun objeto social complejo, polisémico y diverso. Se trata en definitiva, como propone Lupicinio Iñíguez (2006, p. 100), de cambiar la perspectiva de nuestra interrogación prescindiendo de lo dado por descontado y asumiendo una postura problematizadora «que permita abrir nuevas perspectivas de estudio y haga emerger nuevos objetos de investigación».

En línea con esta argumentación consideramos la postura de la Educación Física y el deporte ante las personas mayores. Se trata de una relación mediatizada por las RSs que los agentes sociales poseen sobre la vejez, el cuerpo, la salud y el deporte, no exentas de estereotipos y prejuicios que condicionan las prácticas sociales. Constituye un problema social y a la vez un reto para las instituciones erradicar las prácticas sociales a que dan lugar, denunciando estos contenidos de las RSs sobre la vejez y actividades físico deportivas por ejemplo desde el Sistema Educativo y los medios de comunicación, y sobre todo favoreciendo las condiciones estructurales necesarias para el cambio social.

Una orientación sobre la función social que debería desempeñar el deporte en relación con las personas mayores nos la ofrece la definición de calidad de vida que propone la OMS «percepción que un individuo tiene de su lugar en la existencia, en el contexto de la cultura y del sistema de valores en los que vive y en relación con sus expectativas, sus normas, sus inquietudes». Se trata de un concepto muy amplio que está influido de modo complejo por la salud física del sujeto, su estado psicológico, sunivel de independencia, sus relaciones sociales, así como su relación con los elementos esenciales de su entorno.

\section{Bibliografía}

Allport, G. W. (1977). La naturaleza del prejuicio. Buenos Aires: Editorial Universitaria de Buenos Aires (EUDEMA)

Araya Umaña, S. (2002). Las representaciones sociales: ejes teóricos para su discusión. (L. Villegas, Ed.) Cuaderno de Ciencias Sociales (127).

Ariño, A. (2007). Ideología, sistemas de creencias y representaciones sociales. En J. Cerrato, \& A. Palmonari, Representaciones sociales y psicología social: Comportamiento, globalización y posmodernidad (págs. 138-153). Valencia: Promolibro.
Banchs, M. A. (2000). Aproximaciones procesuales y estructurales al estudio de las Representaciones Sociales. Papers on Social Representations. Textes sur les représentations sociales , 9, 3.1-3.15.

Bourdieu, P. (1998). La distinción. Criterio y bases sociales del gusto. (ed. orig. 1979). Madrid: Taurus.

Brohm, J. M. (1993). 20 Tesis sobre el deporte. En J. M. Brohm, Materiales de sociología del deporte (págs. 47-55). Madrid: La Piqueta.

Brohm, J. M. (1978). Sociología política del deporte. En J. M. Brohm, Partisans: Deporte, cultura y represión (págs. 17-31). Barcelona: Gustavo Gilli.

Cagigal, J. M. (1981). ¡Oh deporte; (Anatomía de un gigante). Valladolid: Miñón.

Cano Gestoso, J. I. (1993). Los estereotipos sociales: el proceso de perpetuación a traves de la memoria selectiva. (Tesis doctoral). Madrid: Universidad complutense.

Cerrato, J., \& Villarreal, M. (2007). Representaciones sociales: Historia, Teoría y Método. En J. Cerrato, \& A. Palmonari, Representaciones sociales y psicología social: Comportamiento, globalización y posmodernidad (págs. 40-116). Valencia: Promolibro.

Coubertin, P. (1973). Ideario Olímpico. Madrid: Instituto Nacional de Educación Física.

Deconchy, J. P. (1986). Sistemas de creencias y representaciones ideológicas. En S. Moscovici, Psicología social II: Pensamiento y vida social. Psicología social y problemas sociales (págs. 439-467). (Ed.orig.1984). Barcelona: Paidós.

Durántez Corral, C. (2002). Filosofía y pedagogía del Olismpismo. Congreso Internacional Historia de la Educación Física Salamanca 16-18 de mayo de 2002 (págs. 1524). Madrid: Gymnos.

Durantez Corral, C. (1975). Olimpia y los Juegos Olímpicos antiguos. Pamplona: Delegación Nacional de Educación Física y Deportes/Comité Olímpico Español.

Elias, N., \& Dunning, E. (1992). Deporte y ocio en el proceso de la civilización. Madrid: Fondo de Cultura Económica.

García Ferrando, M. (1990). Aspectos sociales del deporte. Una reflexión sociológica. Madrid: Alianza Editoria.

Garrido, A., \& Álvaro, J. L. (2007). Psicología social. Perspectivas psicológicas y sociológicas (Segunda edición ed.). Madrid: Mc Graw Hill.

Hernández Mendo, A. (1999). Acerca del término Deporte. Recuperado el 10 de Octubre de 2005, de Educación Física y Deportes nº 17: http://www.efdeportes.com/efd17/ deporte.htm

Ibáñez, T. (2001). Municiones para disidentes. Realidad-Verdad-Política. Barcelona: Gedisa.

Iñíguez Rueda, L. (2006). Análisis del Discurso. Manual para las ciencias sociales. Barcelona: UOC.

Jodelet, D. (1986). La representación social: Fenómenos, concepto y teoría. En S. Moscovici, Psicología social II: Pensamiento y vida social. Psicología social y problemas sociales (págs. 469-494). (Ed.orig.1984). Barcelona: Paidós.

Lippmann, W. (2003). La opinión pública. Madrid: Langre.

Londoño Zapata, O. I. (2007). El análisis crítico del discurso (ACD): una actitud de resistencia [entrevista a Teum van Dijk). Revista Latinoamericana de Estudios del Discurso , 6 (1), 129-135.

Lüschen, G., \& Weis, K. (1979). Sociología del Deporte. Valladolid: Ed. Miñón.

Meynaud, J. (1972). El deporte y la política: análisis social de unas relaciones ocultas. Barcelona: Hispano Europea.

Molliner, P. (1996). Images et représentations sociales. Grenoble: Presses Universitaires de Grenoble.

Moñivas, A. (1998). Representaciones de la vejez (modelos de disminución y de crecimiento). Anales de Psicología , 14 (1), 13-25.

Moscovici, S. (1999). Psicología social I: Influencia y cambio de actitudes .Individuos y grupos. (ed.orig.1984). Barcelona: Paidós.

Moscovici, S. (1986). Psicología social II: Pensamiento y vida social. Psicología social y problemas sociales. (ed.orig.1984). Barcelona: Paidós.

Moscovici, S., \& Pérez, J. A. (1997). Representations of society and prejudices. Papers on Social Representations. Textes sur les Représentations sociales , 6 (1), 27-36.

Pardo Abril, N. G (2007). Niveles de organización del significado en el discurso. Discurso \& sociedad , 1 (1), 85-115

Parlebás, P. (1988). Elementos de Sociología del Deporte. Málaga: Junta de Andalucía/ Universidad Internacional Deportiva de Andalucía.

Rodríguez Cerda, Ó. (2003). Las representaciones sociales: entretejidos de la razón y la cultura. Relaciones , XXIV (93), 83-95.

Rodríguez Salazar, T., \& García Curiel, M. L. (2007). Representaciones sociales. Teoría e investigación. Guadalajara, México: CUCSH-UDG.

Roussiau, N., \& Renard, E. (2003). Des représentations sociales á l'institutionnalisation de la mémoire sociale. Connexions (80), 31-41.

Ruiz Cazorla, L. J. (16 de Mayo de 2008). Función social del deporte espectáculo: las Carreras de Carros en la Antigua Roma (Tesis Doctoral). Málaga: Universidad de Málaga.

Ruiz Cazorla, L. J., \& Chinchilla Minguet, J. L. (2008). Función social del deporte I. Marco teórico. II Congreso Internacional de Ciencias del Deporte. Pontevedra: Alto Rendimiento.

Sánchez Ferlosio, R. (31 de Mayo de 1997). El deporte y el Estado. El País .

Silva, O. (2002). El análisis del discurso según Van Dijk y los estudios de la comunicación. Razón y palabra (26).

Tajfel, H. (1984). Grupos humanos y categorías sociales. (Ed. orig. 1981). Barcelona: Editorial Erder.

Van Dijk, T. (1996). Análisis del discurso ideológico. Versión (6), 15-43.

Van Dijk, T. (1999). El análisis crítico del discurso. Anthropos , 186, 23-26.

Van Dijk, T. (2001). El análisis crítico del discurso y el pensamiento social. Athenea Digital (1), 18-24.

Viaud, J. (2003). Mémoire collective, représentations sociales et pratiques sociales. Connexions (80), 14-30. 